\title{
Pulmonale Effekte auf das synthetisch hergestellte Lipopeptid MALP-2: In-vivo- und In-vitro- Untersuchungen zur Charakterisierung der Leuko- zytenakkumulation in der Rattenlunge
}

\section{A. Lührmann}

\author{
Pulmonary Effect of the Synthetic Lipopeptide MALP-2: In Vivo and in Vitro \\ Studies to Characterize the Leukocyte Accumulation in the Rat Lung
}

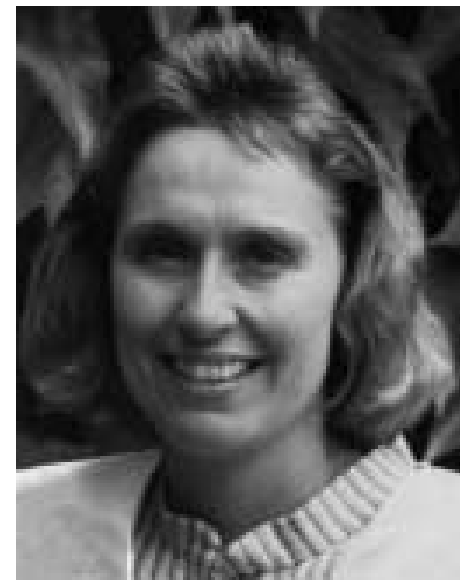

Dr. rer. nat. Anke Lührmann

Die Lunge ist mit ihrer großen Schleimhautoberfläche der Eintrittsort vieler Antigene.

Untersuchungen in der humanen Lunge müssen sich in der Regel aus ethischen Gründen auf die Zellen des bronchoalveolären Raumes, die durch eine Lavage (BAL) gewonnen werden können, und auf Lungengewebe, das nach operativen Eingriffen oder Obduktionen zur Verfügung steht, beschränken. Im Tiermodell ist es dagegen möglich und sinnvoll, die Lunge in verschiedene Kompartimente einzuteilen (Pabst und Tschernig, 1995 Anat Embryol). Hierbei kann zwischen dem Bronchialepithel, dem bronchoalveolären Raum, dem bronchialen Gefäßbett (marginaler
Pool) und dem Lungeninterstitium unterschieden werden. Bei wenigen Spezies findet sich zusätzlich zu den genannten Kompartimenten das so genannte bronchusassoziierte lymphatische Gewebe in der Schleimhaut (Pabst und Gehrke, 1990 Am J Resp Cell Biol). Ähnlich wie den Peyer-Plaques im Darm wird ihm eine wichtige Rolle bei der Aufnahme von Antigenen zugeschrieben. Es gibt wenig Daten darüber, inwieweit die Zellen zwischen den verschiedenen Kompartimenten migrieren können. Obwohl Untersuchungen im Tiermodell in den letzten Jahren Aufschluss über Immunreaktionen in der Lunge gegeben haben, bleiben viele Aspekte zum Auslösen und zur Regulation einer Immunantwort noch ungeklärt. Die Ergebnisse dieser Arbeit geben einige neue Erkenntnisse zur Initiation und Charakterisierung einer Zellakkumulation in der Lunge, wie sie bei Infektionen oder Allergien beobachtet wird.

Für die Experimente stand das ursprünglich aus einem $\mathrm{Myco-}$ plasma-fermentans-Klon isolierte, nun synthetisch hergestellte Lipopeptid MALP-2 (macrophage-activating lipopeptide of molecular mass $2 \mathrm{kDa}$ ) zur Verfügung (Mühlradt u. Mitarb., J Exp Med 1997). In früheren Untersuchungen konnte gezeigt werden, dass MALP-2 in der Lage ist, über die Aktivierung der Toll-like-Rezeptoren (TLR) 2 und 6 (Takeuchi u. Mitarb., Immunity 1999, J Immunol 2000), Makrophagen zu aktivieren. Im In-vivo-Mausmodell haben Deiters und Mühlradt (Inf Imm 1999) nach intraperitonealer Applikation von MALP-2 eine stark aktivierende Wirkung auf Makrophagen unter Ausschüttung verschiedener Chemokine wie MCP-1, MIP-1 $\alpha$ und MIP-1 $\beta$ nachweisen können.

Institutsangaben

Med. Hochschule Hannover

Anmerkung

Die Arbeit wurde durch die Deutsche Forschungsgemeinschaft (Pa 240/8-2) gefördert.

Das Lipopeptid MALP-2 wurde freundlicherweise von Prof. P. F. Mühlradt, GBF Braunschweig

zur Verfügung gestellt. Die Autorin dankt all denen ganz herzlich, die bei der Erstellung dieser Arbeit unterstützend mitgewirkt haben.

Korrespondenzadresse

Dr. rer. nat. Anke Lührmann - aus der Arbeitsgruppe von Prof. Dr. med. Reinhard Pabst . Abteilung Funktionelle und Angewandte Anatomie - Medizinische Hochschule Hannover .

Carl-Neuberg-Str. $1 \cdot 30323$ Hannover

Bibliografie

Pneumologie 2003; 57: 468-469 @ Georg Thieme Verlag Stuttgart · New York · ISSN 0934-8387 
In der vorliegenden Arbeit konnte die Wirkung von MALP-2 in der Lunge durch durchflusszytometrische, histologische und molekularbiologische Untersuchungsmethoden in verschiedener Hinsicht charakterisiert werden. Die lokale Applikation von MALP-2 führte zu einer dosis- und zeitabhängigen Akkumulation verschiedener Leukozytenpopulationen in der Lunge. Die Zellzahlerhöhungen in den verschiedenen Kompartimenten der Rattenlunge unterschieden sich deutlich voneinander. Waren im Lungeninterstitium und im bronchialen Gefäßbett die Zellzahlen nur bei einigen Zellpopulationen angestiegen, so kam es zu einem dramatischen Anstieg fast aller untersuchten Zellpopulationen im bronchoalveolären Raum. Dass diese Wirkung durch einen lokalen Effekt erzielt wurde, konnte durch die intraperitoneale Gabe des MALP-2 gezeigt werden. Hierbei kam es im Vergleich zur intratrachealen Applikation zu einem wesentlich geringeren Zellzahlanstieg in der Lunge.

Bei der intratrachealen Behandlung von jungen, entwöhnten Ratten war die Zellzahl im Vergleich zu adulten, 8-10 Wochen alten Ratten in der BAL mit Ausnahme der T-Lymphozyten nicht höher. Überraschend war dagegen, dass die Zellzahlen im Lungeninterstitium bei den jungen Tieren in allen untersuchten Zellpopulationen signifikant höher waren.

In-vitro-Versuche zeigten, dass die Stimulation von Alveolarmakrophagen mit MALP-2 zu einer schnellen und kurz dauernden Erhöhung der Expression der mRNA für den TLR2 führt. Die These, dass die Aktivierung über den TLR vermutlich zu einer Änderung des Mikromilieus in der Lunge führt, konnte durch den Nachweis der Erhöhung der chemotaktischen Aktivität und des Chemokins MCP-1 im Überstand der BAL sowie im Lungengewebe bekräftigt werden.

In Inhalationsversuchen mit MALP-2 über mehrere Wochen konnte die Zunahme von bronchusassoziiertem lymphatischen Gewebe in der Rattenlunge dokumentiert werden.

In einem Modell für eine Metastasierung in der Lunge wurde gezeigt, dass es bei gleichzeitiger Applikation von Tumorzellen und MALP-2 zu einer deutlichen Reduktion der Metastasenzahl kommt.
Zusammenfassend kann festgestellt werden, dass lokal appliziertes MALP-2 ein potenter Immunstimulator für die Lunge ist. In Zukunft können unter Einsatz dieses Lipopeptids grundlegende Mechanismen, die zum Rekruitment von Immunzellen in die Lunge führen, und deren Regulation, untersucht werden. Dieses Grundverständnis wird benötigt, um bei Fehlregulationen des Immunsystems eingreifen zu können.

Weiterhin soll die Wirkung von MALP-2 als Adjuvans getestet werden. Möglicherweise kann ein Organismus, der zuvor gezielt stimuliert worden ist, mit einer verbesserten Immunantwort auf ein Antigen reagieren. Dies würde Vorteile bei verschiedenen Vakzinierungsstrategien bringen.

Möglicherweise könnte MALP-2 nach intensiven Studien in der Tumorbekämpfung eingesetzt werden. Es ist bekannt, dass es bei der operativen Entfernung von Karzinomen häufig zu einer Streuung von Tumorzellen kommt, die bevorzugt in der Lunge „hängen“ bleiben und dort metastasieren. Eine präoperative Gabe von MALP-2 könnte eventuell die Metastasierung der Tumorzellen vermindern oder sogar verhindern, indem aktivierte Immunzellen diese vernichten.

Teile dieser Arbeit wurden veröffentlicht:

In vivo effects of a synthetic 2-kilodalton macrophage-activating lipopeptide of Mycoplasma fermentans after pulmonary application. Lührmann A, Deiters U, Skokowa J, Hanke M, Gessner JE, Mühlradt PF, Pabst R, Tschernig T. Infect Immun 2002; 70: $3785-3792$.

Intratracheal macrophage-activating lipopeptide- 2 reduces metastasis in the rat lung. Shingu K, Kruschinski C, Lührmann A, Grote K, Tschernig T, von Hörsten S, Pabst R. Am J Respir Cell Mol Biol 2003; 28: $316-321$.

Stimulation of bronchus-associated lymphoid tissue in rats by repeated inhalation of aerosolized lipopeptide MALP-2. Lührmann A, Tschernig T, Pabst R. Pathobiology 2002-2003; 70: $266-269$. 